\title{
Risk factors of suicide attempts by poisoning: review
}

\author{
Fatores de risco para tentativa de suicídio por envenenamento: \\ revisão
}

\author{
Maria Cláudia da Cruz Pires, ${ }^{1}$ Tatiana de Paula Santana da Silva, ${ }^{2}$ Marcela Pires dos Passos, ${ }^{3}$ Everton Botelho Sougey ${ }^{4}$ \\ Othon Coelho Bastos Filho ${ }^{5}$
}

\begin{abstract}
Introduction: Suicide, a complex and universal human phenomenon, is a major public health problem. This study reviewed the literature about the major risk factors associated with suicide attempts by poisoning.

Methods: An integrative review of the literature was performed in databases (LILACS, PubMed and MEDLINE) to search for studies published between 2003 and 2013, using the following keywords: suicide, attempted; poisoning; risk factors. Inclusion criteria were: original study with abstract, sample of adults, and attempted suicide by poisoning in at least $50 \%$ of the study population. Results: Two hundred and nineteen studies were retrieved and read by two independent examiners, and 22 were included in the study. The main risk factors for suicide attempts by poisoning were female sex, age 15-40 years, single status, little education, unemployment, drug or alcohol abuse or addiction, psychiatric disorder and psychiatric treatment using antidepressants.

Conclusion: Further prospective studies should be conducted to confirm these risk factors or identify others, and their findings should contribute to planning measures to prevent suicide attempts.
\end{abstract}

Keywords: Suicide, attempt, poisoning, risk factors.

\section{Resumo}

Introdução: $O$ suicídio é um fenômeno humano complexo, universal e que representa um grande problema de saúde pública. Diante disso, o presente estudo tem por objetivo revisar a literatura acerca dos principais fatores de risco associados à tentativa de suicídio por envenenamento.

Método: Foi realizada uma revisão integrativa em bases de dados (LILACS, PubMed e MEDLINE). A pesquisa focou artigos publicados entre os anos de 2003 a 2013, utilizando os seguintes descritores: suicide, attempted; poisoning; risk factors. Foram considerados os seguintes critérios de inclusão: ser artigo original, com resumo disponível, amostra composta por adultos, e que pelo menos $50 \%$ da população do estudo tenham tentado o suicídio por envenenamento.

Resultados: Foram selecionados 219 artigos. Após a leitura por dois pesquisadores independentes, 22 foram incluídos na revisão. Os principais fatores de risco encontrados para a tentativa de suicídio por envenenamento foram: gênero feminino, idade entre 15 a 40 anos, estado civil solteiro, baixa escolaridade, desemprego, abuso/dependência de substancias e/ou álcool, ser acometido por transtorno psiquiátrico e estar em tratamento psiquiátrico com uso de antidepressivo.

Conclusão: Considera-se que pesquisas adicionais, bem como a ampliação de estudos prospectivos, deverão ser realizadas para a confirmação ou identificação de novos fatores de risco. Estas medidas objetivam contribuir para o planejamento e prevenção da tentativa e do suicídio.

Descritores: Tentativa de suicídio, envenenamento, fatores de risco.

\footnotetext{
${ }^{1}$ Psychiatrist. PhD, Graduate Program in Neuropsychiatry and Behavioral Sciences, Universidade Federal de Pernambuco (UFPE), Recife, PE, Brazil. ${ }^{2}$ Speech therapist. Doctoral student, Graduate Program in Neuropsychiatry and Behavioral Sciences, UFPE, Recife, PE, Brazil. ${ }^{3}$ Psychologist. PhD, Graduate Program in Neuropsychiatry and Behavioral Sciences, UFPE, Recife, PE, Brazil. ${ }^{4}$ PhD. Professor, Graduate Program in Neuropsychiatry and Behavioral Sciences, UFPE, Recife, PE, Brazil. 5 Psychiatrist. Full Professor of Psychiatry, UFPE, Recife, PE, Brazil.

This study was carried out at the Graduate Program in Neuropsychiatry and Behavioral Sciences of Universidade Federal de Pernambuco (UFPE), Recife, PE, Brazil. Financial support: Graduate Program in Neuropsychiatry and Behavioral Sciences, Universidade Federal de Pernambuco (UFPE), Recife, PE, Brazil.

Submitted Sep 13 2013, accepted for publication Feb 24 2014. No conflicts of interest declared concerning the publication of this article.

Suggested citation: Pires MC, da Silva TP, dos Passos MP, Sougey EB, Bastos Filho OC. Risk factors of suicide attempts by poisoning: review. Trends Psychiatry Psychother. 2014;36(2):63-74. http://dx.doi.org/10.1590/2237-6089-2013-0044
} 


\section{Introduction}

The comprehension of the definitions of suicide and attempted suicide is based on several theoretical domains, such as theology, psychology, philosophy and sociology, as well as psychiatry. ${ }^{1}$ The World Health Organization $^{2}$ defines suicide as the deliberate act of killing oneself. One of the first definitions of attempted suicide classified it as "any non-fatal act of self-damage inflicted with self-destructive intention, however vague and ambiguous." ${ }^{3}$

In later publications, the objective intention to kill oneself has not been found as an obligatory requisite in the definition of attempted suicide. The term "suicidal behavior" might, therefore, be equivalent to the term "self-harm", and both define "an act of intentional selfpoisoning or injury irrespective of the apparent purpose of the act". ${ }^{4}$ This definition is closer to the terms "attempted suicide" and "parasuicide" used in several countries. ${ }^{4}$

These definitions are, however, imprecise, as it is difficult to evaluate and measure the intention of a desire to die or not die underlying the acts described. ${ }^{5}$ Therefore, despite all efforts, no unanimous and consensual definition of attempted suicide has been reached.

Epidemiological data show that, when compared with rates in other countries, mortality due to suicide in Brazil is one of the lowest, although Brazil is one of the ten countries with the highest absolute number of deaths due to suicide. 6,7 From 2003 to 2009, there were 60,637 deaths due to suicide in Brazil, at a mean 24 deaths per day, which results in a mean coefficient of 4.5 deaths per 100 thousand inhabitants (Departamento de Informática do SUS - Sistema de Informações sobre Mortalidade Brazilian Mortality Information System). ${ }^{8}$ Of all deaths due to external causes $(6.6 \%)$, suicide accounts for $0.8 \%$ of the total in Brazil. ${ }^{9}$

The number of attempted suicides is expected to be higher than that of suicides. For each actual suicide, about 20 to 30 suicidal behaviors are expected, and only about one fourth of them will have any contact with healthcare services. ${ }^{10,11}$ In Brazil, attempted suicides are not compulsorily reported, and reports currently made are rare and less reliable than those made for suicides. ${ }^{12}$ The analysis of suicide attempt methods reveals an association between method used or chosen and the sex of individuals. For example, men prefer potentially lethal methods, such as hanging or shooting, ${ }^{13-15}$ whereas women use less aggressive and slower methods, such as some types of poisoning. ${ }^{16-18}$

The study of suicide attempts by poisoning is justified by the consistent evidence of a growing use of toxic agents as a method. ${ }^{19-22}$ Of the main toxic agents, medications have been increasingly used in the last decades, and are now more frequent than pesticides. For example, in 2006, according to our review, the number of attempted suicides by using medications was greater than twice the number of attempts by using pesticides. When compared with other agents, their numbers are relatively stable from 1994 to $2006 .^{23,24}$

Attempted suicides have been classified as a public health problem because of their reach.9,10,20 There are several explanations for this type of behavior, in addition to easy access to toxic agents, and in some cases there is a clear association with complex psychosocial phenomena, mental disorders and negative life events. ${ }^{18,25}$

Possible risk factors associated with this phenomenon should be studied to support the development of more efficacious preventive strategies. This study reviewed the current literature about the main risk factors associated with attempted suicide by poisoning.

\section{Method}

This integrative literature review was conducted in three phases. In the first, the MEDLINE, LILACS and PubMed databases were chosen for the searches. In the second, the keywords and inclusion criteria to identify studies of interest were defined. Keywords were chosen according to searches conducted in the keyword collections of two of the databases, that is, the Medical Subject Headings (MeSH) and the Descritores em Ciências da Saúde (DeCS, a Brazilian database of medical keywords).

The keywords used to select studies were suicide, attempted, poisoning and risk factors, as well as their equivalent Portuguese terms (tentativa de suicídio, envenenamento, fatores de risco). The Boolean operators AND and AND NOT were used to combine keywords and terms for the publication searches.

The selection was limited to studies published in Portuguese, English or Spanish from January 2003 to March 2013. The last database search was conducted in July 2013.

In the third phase, study titles and abstracts of all the studies selected were read to identify those that dealt with the topic of this review. Additionally, the following inclusion criteria were adopted: original study; and studies in which poisoning was the main method for at least $50 \%$ of the population that attempted suicide. Studies were excluded if published as editorials, interviews, clinical notes or reviews, as well as those with samples that included only children, adolescents or the elderly.

The initial search retrieved 222 potentially eligible studies: 180 in PubMed, 37 in MEDLINE and 5 in LILACS. They were read independently by two examiners. When they disagreed, a third examiner was asked to give an 
opinion about whether the study should be included or excluded. At the end of those analyses, 22 studies were included in this review.

The flowchart in Figure 1 describes the literature review and the selection of studies.

Table 1 shows the characteristics of the studies included in this review. A greater number of studies were published from 2010 to 2012. Most studies were retrospective, followed by multi-center studies. These results indicate that the study of situations with an imminent risk of death, such as attempted suicide, is a difficult task, although necessary for the understanding of the problem and the planning of more efficient preventive measures. ${ }^{26,45}$

This extensive review of risk factors found that some studies reported an incidence of attempts by self-poisoning greater than 50\%. However, only the risk factors exclusively associated with poisoning are discussed here, as shown in Table 1 , in the column for attempt method.

The impact factor of each publication was analyzed to classify studies according to level of evidence. Of the 22 studies, 10 were published in journals with an impact factor greater than 1.0. This criterion is a measure used in bibliometrics to evaluate the quality of a journal in terms of citations and access and provides, therefore, one of the best indices of evidence for any topic. ${ }^{46}$

Most of the studies in this review were conducted in Europe, followed by Asia, and most included samples with more than 900 individuals.

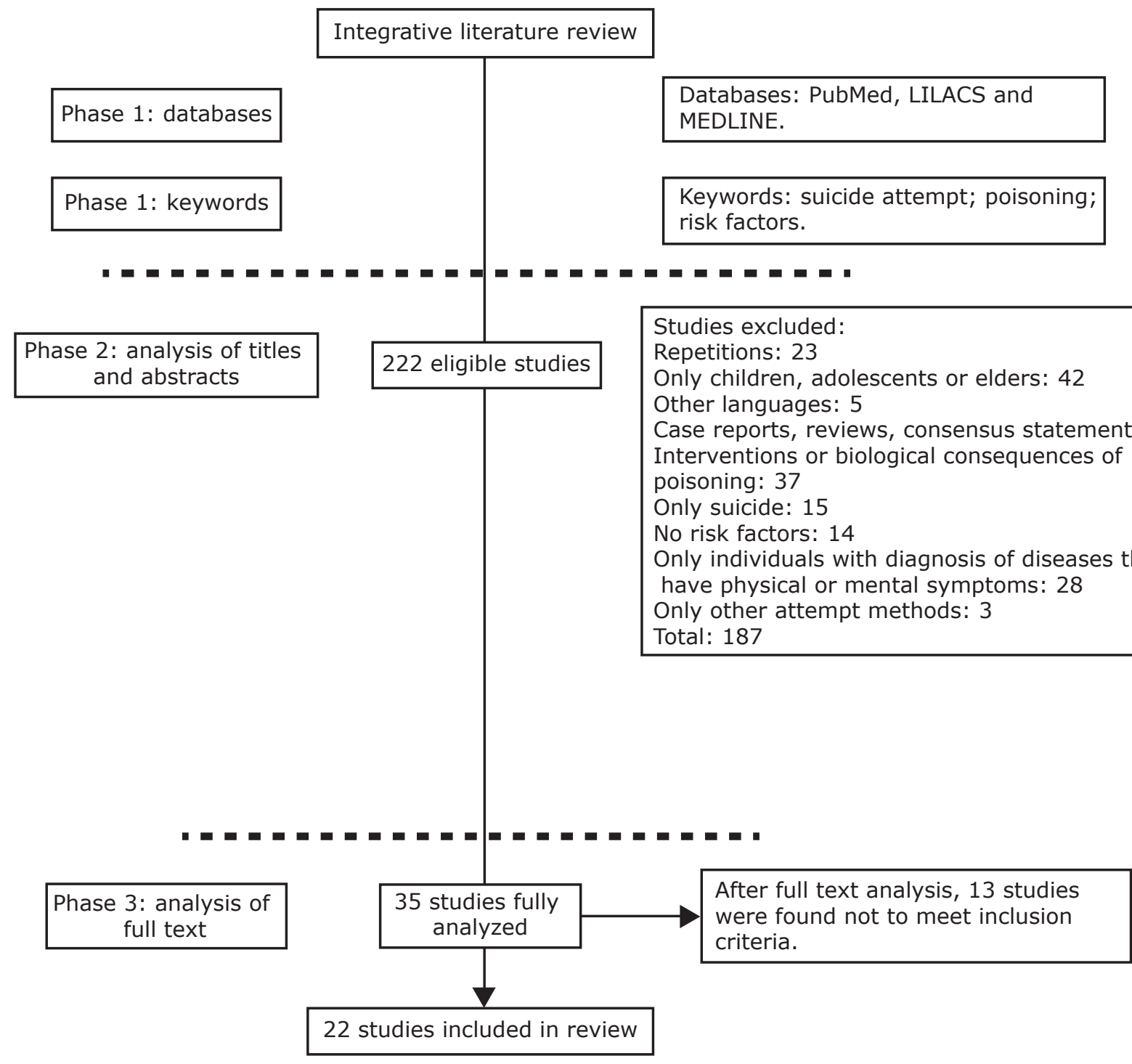

Figure 1 - Literature review flowchartResults and discussion 
Table 1 - Characteristics of the studies included in the literature review

\begin{tabular}{|c|c|c|c|c|c|}
\hline $\begin{array}{l}\text { Authors, year and } \\
\text { impact factor }\end{array}$ & Type of study & $\begin{array}{l}\text { Place and } \\
\text { country }\end{array}$ & $\begin{array}{l}\text { Study duration and } \\
\text { sample size }\end{array}$ & Sex and age & Attempt method \\
\hline $\begin{array}{l}\text { Flavio et al., } 2013^{26} \\
\text { Swiss Med Wkly } \\
\text { Impact factor: } 1.895\end{array}$ & Comparative & $\begin{array}{l}\text { Basel City, } \\
\text { Switzerland }\end{array}$ & $\begin{array}{l}35 \text { months (Jan } 2003 \\
\text { to Dec 2006) } \\
\text { Sample: } 778\end{array}$ & $\begin{array}{l}\text { Men: } 326(33 \%) \\
\text { Women: } 658(67 \%) \\
\text { Age: } 15 \text { to } 103 \text { years }\end{array}$ & $\begin{array}{l}\text { Poisoning: more than } \\
50 \%\end{array}$ \\
\hline $\begin{array}{l}\text { Williams-Jonshon et } \\
\text { al., } 2012^{27} \\
\text { West Indian* }\end{array}$ & Retrospective & $\begin{array}{l}\text { Kingston, } \\
\text { Jamaica }\end{array}$ & $\begin{array}{l}48 \text { months (2005- } \\
2009 \text { ) } \\
\text { Sample: } 127\end{array}$ & $\begin{array}{l}\text { Men: } 31(24.4 \%) \\
\text { Women: } 96(75.6 \%) \\
\text { Age: } 10 \text { to } 55 \text { years }\end{array}$ & All by poisoning \\
\hline $\begin{array}{l}\text { Rancic et al., } 2012^{28} \\
\text { Med Glas } \\
\text { Impact factor: } 0.056\end{array}$ & Retrospective & $\begin{array}{l}\text { Kragujevac, } \\
\text { Serbia }\end{array}$ & $\begin{array}{l}240 \text { months (1990- } \\
2010) \\
\text { Sample: } 715\end{array}$ & $\begin{array}{l}\text { Men: } 310(43.5 \%) \\
\text { Women: } 405(56.5 \%) \\
\text { Age: } 15 \text { to } \geq 65 \text { years }\end{array}$ & $\begin{array}{l}\text { Poisoning: } 395(55.3 \%) \\
\text { Hanging: } 63(8.8 \%)\end{array}$ \\
\hline $\begin{array}{l}\text { Lee et al., } 2012^{29} \\
\text { J Korean Med } \\
\text { Impact factor: } 0.993\end{array}$ & Cross-sectional & Suwon, Korea & $\begin{array}{l}36 \text { months (2007- } \\
2009) \\
\text { Sample: } 2996\end{array}$ & $\begin{array}{l}\text { Men: } 1259(42 \%) \\
\text { Women: } 1737(58 \%) \\
\text { Age: } 10 \text { to } \geq 60 \text { years }\end{array}$ & $\begin{array}{l}\text { Poisoning by medication } \\
\text { overdose } 68.8 \% \\
\text { Other methods: } 28,2 \%\end{array}$ \\
\hline $\begin{array}{l}\text { Turhan et al., } 2012^{30} \\
\text { Neurosciences } \\
\text { (Riyadh) } \\
\text { Impact factor: } 0.121\end{array}$ & $\begin{array}{l}\text { Retrospective } \\
\text { (historical } \\
\text { cohort) }\end{array}$ & Hatay, Turkey & $\begin{array}{l}36 \text { months (2007- } \\
2009) \\
\text { Sample: } 1.613\end{array}$ & $\begin{array}{l}\text { Men: } 343(21.1 \%) \\
\text { Women: } 1270(78.9 \%) \\
\text { Age: } 10 \text { to } 63 \text { years }\end{array}$ & $\begin{array}{l}\text { Poisoning by medication } \\
\text { overdose } 97.5 \% \\
\text { Other methods: } 2,5 \%\end{array}$ \\
\hline $\begin{array}{l}\text { Garcia-Rabago et al., } \\
2010^{31} \\
\text { Rev Salud Pub* }\end{array}$ & $\begin{array}{l}\text { Prospective, } \\
\text { observational, } \\
\text { comparative }\end{array}$ & Jalisco, Mexico & $\begin{array}{l}6 \text { months (2005) } \\
\text { Sample: } 106\end{array}$ & $\begin{array}{l}\text { Men: } 32(30.2 \%) \\
\text { Women: } 1270(78.9 \%) \\
\text { Age: } 15 \text { to } 61 \text { years }\end{array}$ & $\begin{array}{l}\text { Poisoning by medication } \\
\text { overdose }(60.3 \%)\end{array}$ \\
\hline $\begin{array}{l}\text { Yip et al., } 2011^{32} \\
\text { Crisis* }\end{array}$ & Comparative & $\begin{array}{l}\text { Hong Kong } \\
\text { and Oxford }\end{array}$ & $\begin{array}{l}3 \text { months (2005) } \\
\text { Sample: } 90\end{array}$ & $\begin{array}{l}\text { Men: } 26(29 \%) \\
\text { Women: } 64(71 \%) \\
\text { Age: mean age: } 35 \\
\text { years }\end{array}$ & $\begin{array}{l}\text { Poisoning by medication } \\
\text { ingestion }(78 \%)\end{array}$ \\
\hline $\begin{array}{l}\text { Oh et al., } 2011^{22} \\
\text { Am J Emerg Med } \\
\text { Impact factor: } 1.976\end{array}$ & Retrospective & Seoul, Korea & $\begin{array}{l}\text { 2000-2009 } \\
\text { Sample: } 967\end{array}$ & $\begin{array}{l}\text { Men: } 220(22.8 \%) \\
\text { Women: } 747(77.3 \%) \\
\text { Age: } 13 \text { to } 94 \text { years }\end{array}$ & All by poisoning \\
\hline $\begin{array}{l}\text { Rezaie et al., } 2011^{33} \\
\text { Burns } \\
\text { Impact factor: } 1.962\end{array}$ & $\begin{array}{l}\text { Intervention; } \\
\text { quasi- } \\
\text { experimental }\end{array}$ & $\begin{array}{l}\text { Kermanshah, } \\
\text { Iran }\end{array}$ & $\begin{array}{l}3 \text { months (Jun to Sept } \\
\text { 2006) } \\
\text { Sample: } 200\end{array}$ & $\begin{array}{l}\text { Men: } 67(34 \%) \\
\text { Women: } 133(63 \%) \\
\text { Age: } 26.4 \pm 9.5 \text { years }\end{array}$ & $\begin{array}{l}\text { Poisoning: } 137(68 \%) \\
\text { Immolation } 63(32 \%)\end{array}$ \\
\hline $\begin{array}{l}\text { Chowdhury et al., } \\
2010^{34} \\
\text { Natl Med J indian } \\
\text { Impact factor: } 0.595\end{array}$ & Prospective & Bengal, India & $\begin{array}{l}12 \text { months (2002) } \\
\text { Sample: } 1614\end{array}$ & $\begin{array}{l}\text { Men: } 619(38.4 \%) \\
\text { Women: } 995(61.7 \%) \\
\text { Age: } 7 \text { to } 80 \text { years }\end{array}$ & $\begin{array}{l}\text { Poisoning: } 1588 \\
(98.4 \%) \\
\text { Other methods: } 1.6 \%\end{array}$ \\
\hline $\begin{array}{l}\text { Zyoud et al., } 2010^{24} \\
\text { Hum } \\
\text { Psychopharmacol* }\end{array}$ & $\begin{array}{l}\text { Descriptive and } \\
\text { retrospective }\end{array}$ & Malaysia, Asia & $\begin{array}{l}36 \text { months (2006- } \\
\text { 2008) } \\
\text { Sample: } 177\end{array}$ & $\begin{array}{l}\text { Men: } 28(15.8 \%) \\
\text { Women: } 149(84.2 \%) \\
\text { Age: }<20 \text { to }>30\end{array}$ & $\begin{array}{l}\text { All by poisoning } \\
\text { with acetaminophen } \\
\text { overdose }\end{array}$ \\
\hline $\begin{array}{l}\text { Heyerdahl et al., } \\
2010^{35} \\
\text { BMC Psychiatry } \\
\text { Impact factor: } 2.552\end{array}$ & $\begin{array}{l}\text { cohort } \\
\text { prospective } \\
\text { multi-site }\end{array}$ & Oslo, Norway & $\begin{array}{l}11 \text { months (2003- } \\
\text { 2004) } \\
\text { Sample: } 908\end{array}$ & $\begin{array}{l}\text { Men: } 418(46 \%) \\
\text { Women: } 490(54 \%) \\
\text { Age: }<16-89 \text { years }\end{array}$ & All by poisoning \\
\hline $\begin{array}{l}\text { Ozdel et al., } 2009^{36} \\
\text { Crisis* }\end{array}$ & Cross-sectional & $\begin{array}{l}\text { Pamukkale, } \\
\text { Turkey }\end{array}$ & $\begin{array}{l}24 \text { months (2006- } \\
2007 \text { ) } \\
\text { Sample: } 144\end{array}$ & $\begin{array}{l}\text { Men: } 36(25 \%) \\
\text { Women: } 108(75 \%) \\
\text { Age: }<16-89 \text { years }\end{array}$ & $\begin{array}{l}\text { Poisoning by } \\
\text { medication: } 70 \% \\
\text { Other methods: } 30 \%\end{array}$ \\
\hline $\begin{array}{l}\text { Payne et al., } 2009^{37} \\
\text { Public Health } \\
\text { Impact factor: } 1.35\end{array}$ & $\begin{array}{l}\text { Retrospective } \\
\text { cohort }\end{array}$ & $\begin{array}{l}\text { Scotland, } \\
\text { United } \\
\text { Kingdom }\end{array}$ & $\begin{array}{l}84 \text { months (1996- } \\
2002) \\
\text { Sample: } 50.891\end{array}$ & $\begin{array}{l}\text { Men: } 21883(43 \%) \\
\text { Women: } 29007(57 \%) \\
\text { Age: } 15 \text { to }>65 \text { years }\end{array}$ & All by poisoning \\
\hline $\begin{array}{l}\text { Carter et al., } 2007^{38} \\
\text { Suicide Life Threat } \\
\text { Behav* }\end{array}$ & Case-control & Australia & $\begin{array}{l}44 \text { months (1998- } \\
\text { 2001) } \\
\text { Sample: } 581\end{array}$ & $\begin{array}{l}\text { Men: } 180(31 \%) \\
\text { Women: } 401(69 \%) \\
\text { Age: } 18 \text { to }>65 \text { years }\end{array}$ & All by poisoning \\
\hline $\begin{array}{l}\text { Kapur et al., } 2006^{4} \\
\text { J Clin Psychiatry } \\
\text { Impact factor: } 5.799\end{array}$ & $\begin{array}{l}\text { Prospective } \\
\text { cohort } \\
\text { Multi-site }\end{array}$ & $\begin{array}{l}\text { United } \\
\text { Kingdom }\end{array}$ & $\begin{array}{l}60 \text { months (1997- } \\
2002) \\
\text { Sample: } 9.213\end{array}$ & $\begin{array}{l}\text { Men: } 3991(43 \%) \\
\text { Women: } 5222(57 \%) \\
\text { Age: older than } 15 \\
\text { years }\end{array}$ & $\begin{array}{l}\text { Poisoning: } 87 \% \\
\text { Other methods: } 15 \%\end{array}$ \\
\hline $\begin{array}{l}\text { Carter et al., } 2006^{39} \\
\text { Suicide Life Threat } \\
\text { Behav* }\end{array}$ & $\begin{array}{l}\text { Comparative } \\
\text { cohort }\end{array}$ & $\begin{array}{l}\text { Newcastle, } \\
\text { Australia }\end{array}$ & $\begin{array}{l}60 \text { months (1996- } \\
2002) \\
\text { Sample: } 2629\end{array}$ & $\begin{array}{l}\text { Men: } 991 \text { men }(38 \%) \\
\text { Women: } 1638(62 \%) \\
\text { Age: }<25 \text { to > } 35 \\
\text { years }\end{array}$ & All by poisoning \\
\hline $\begin{array}{l}\text { Alaghehbandan et al., } \\
2005^{40} \\
\text { Can Psychiat } \\
\text { Impact factor: } 2.417\end{array}$ & $\begin{array}{l}\text { Secondary } \\
\text { database; } \\
\text { retrospective }\end{array}$ & $\begin{array}{l}\text { Newfoundland } \\
\text { and Labrador, } \\
\text { Canada }\end{array}$ & $\begin{array}{l}24 \text { months (1998- } \\
2000) \\
\text { Sample:978 }\end{array}$ & $\begin{array}{l}\text { Men: } 423(43 \%) \\
\text { Women: } 550(57 \%) \\
\text { Age: } 10 \text { to } \geq 75 \text { years }\end{array}$ & $\begin{array}{l}\text { Poisoning: } 87.0 \% \text {. } \\
\text { Cutting: } 7.1 \% \\
\text { Hanging: } 2.0 \%\end{array}$ \\
\hline
\end{tabular}


(cont.)

\begin{tabular}{|c|c|c|c|c|c|}
\hline $\begin{array}{l}\text { Ichimura et al., } \\
2005^{41} \\
\text { Psychiatry Clin } \\
\text { Neurosci } \\
\text { Impact factor: } 2.133\end{array}$ & Case-control & Isehara, Japan & $\begin{array}{l}12 \text { months (Jan to Dec } \\
2000 \text { ) } \\
\text { Sample: } 235\end{array}$ & $\begin{array}{l}\text { Men: } 104(44 \%) \\
\text { Women: } 131(56 \%) \\
\text { Age: } 49.2 \text { years }( \pm 16.9)\end{array}$ & $\begin{array}{l}\text { Poisoning: } 172(70 \%) \\
\text { Other methods: } 73 \\
(30 \%)\end{array}$ \\
\hline $\begin{array}{l}\text { Fekete et al., } 2005^{42} \\
\text { Croat Med J } \\
\text { Impact factor: } 1.796\end{array}$ & $\begin{array}{l}\text { Multi-site; } \\
\text { retrospective }\end{array}$ & $\begin{array}{l}\text { Hungary, } \\
\text { Romania }\end{array}$ & $\begin{array}{l}48 \text { months (1997- } \\
2001) \\
\text { Sample: } 1.158\end{array}$ & $\begin{array}{l}\text { Men: } 430(37 \%) \\
\text { Women: } 728(63 \%) \\
\text { Age: } 36.4 \text { years }( \pm 15.2)\end{array}$ & All by poisoning \\
\hline $\begin{array}{l}\text { Douglas et al., } 2004^{43} \\
\text { J Affect Disorders } \\
\text { Impact factor: } 3.517\end{array}$ & Case-control & $\begin{array}{l}\text { Saltford, } \\
\text { United } \\
\text { Kingdom }\end{array}$ & $\begin{array}{l}18 \text { months (Mar } 1999 \\
\text { to Aug 2000) } \\
\text { Sample: } 1748\end{array}$ & $\begin{array}{l}\text { Men: } 710(6 \%) \\
\text { Women: } 1038(94 \%) \\
\text { Age: suicide group: } 41 ; \\
\text { NFDSH group: } 34 ; \text { DSH } \\
\text { group: } 31\end{array}$ & $\begin{array}{l}\text { Poisoning: } 74 \% \\
\text { Other methods: } 26 \%\end{array}$ \\
\hline $\begin{array}{l}\text { Carter et al., } 2003^{44} \\
\text { Aust NZJ Psychiatry } \\
\text { Impact factor: } 2.929\end{array}$ & Case-control & $\begin{array}{l}\text { Newcastle, } \\
\text { Australia }\end{array}$ & $\begin{array}{l}26 \text { months (Apr } 1998 \\
\text { to Jun 2000) } \\
\text { Sample: } 82\end{array}$ & $\begin{array}{l}\text { Men: } 23(28 \%) \\
\text { Women: } 59(72 \%) \\
\text { Age: } 18 \text { to } 24 \text { years }\end{array}$ & All by poisoning \\
\hline
\end{tabular}

* Journal with no impact factor.

Table 2 - Summary of studies that describe risk factors of attempted suicide by poisoning according to objective, risk factors, conclusions and general comments

\begin{tabular}{|c|c|c|c|c|}
\hline Authors, year & Study objective & Risk factors & Conclusions & Comments \\
\hline $\begin{array}{l}\text { Flavio et al., } \\
2013^{26}\end{array}$ & $\begin{array}{l}\text { To analyze rate of attempted } \\
\text { suicides and their methods and to } \\
\text { identify risk groups of attempted } \\
\text { suicide. }\end{array}$ & $\begin{array}{l}\text { Women aged } 37.43 \pm 16.87 \text { ( } p \\
\leq 0.001 \text { ) } \\
\text { Risk groups include single } \\
\text { individuals, of foreign } \\
\text { nationality, low education and } \\
\text { low employment status }\end{array}$ & $\begin{array}{l}\text { Men were significantly } \\
\text { more frequently } \\
\text { affected by substance } \\
\text { abuse disorder or } \\
\text { psychosis, whereas } \\
\text { in women adjustment } \\
\text { disorders and } \\
\text { personality disorders } \\
\text { were diagnosed } \\
\text { significantly more } \\
\text { often. }\end{array}$ & $\begin{array}{l}\text { This study offers } \\
\text { the first published } \\
\text { representative data } \\
\text { of an entire Swiss } \\
\text { county. Established } \\
\text { sociodemographic and } \\
\text { clinical risk factors } \\
\text { for suicide attempts } \\
\text { were reproduced. The } \\
\text { identification of risk } \\
\text { factors contributes } \\
\text { to the development } \\
\text { of local targeted } \\
\text { prevention strategies, } \\
\text { for example education } \\
\text { of risk groups and } \\
\text { caregivers, and } \\
\text { pharmacolegal } \\
\text { consequences for } \\
\text { package sizes. } \\
\text { Gender- and age- } \\
\text { specific prevention and } \\
\text { aftercare programs are } \\
\text { indicated. }\end{array}$ \\
\hline $\begin{array}{l}\text { Williams } \\
\text { Jonshon et al., } \\
2012^{27}\end{array}$ & $\begin{array}{l}\text { To document } \\
\text { the characteristics of self- } \\
\text { poisoning suicide attempters who } \\
\text { were brought to the Emergency } \\
\text { Room of the University Hospital of } \\
\text { the West Indies (UHWI) and to } \\
\text { outline the type of drug used in } \\
\text { the attempt. }\end{array}$ & $\begin{array}{l}\text { Women }(p<0.001) \\
\text { Age } 16 \text { to } 30 \text { years }(p< \\
0.001) \\
\text { Use of chemical agents } \\
\text { greater among women ( } p< \\
0.019)\end{array}$ & $\begin{array}{l}\text { Findings consistent } \\
\text { with global trends of } \\
\text { attempted suicides } \\
\text { according to sex and } \\
\text { age group. }\end{array}$ & $\begin{array}{l}\text { Substance ingested } \\
\text { different from other } \\
\text { data in the literature } \\
\text { (women: chemicals; } \\
\text { men: medication) }\end{array}$ \\
\hline $\begin{array}{l}\text { Rancic et al., } \\
2012^{28}\end{array}$ & $\begin{array}{l}\text { To determine the frequency and } \\
\text { distribution of suicide attempts } \\
\text { relating to the manner of } \\
\text { execution and (...) possible risk } \\
\text { factors. }\end{array}$ & $\begin{array}{l}\text { Women: } 405(56.5 \%) \\
\text { Depression: } 336(48.4 \%) \\
\text { Mean age at attempt: } 42.6 \\
(\mathrm{SD}=16.78) \\
\text { Schizophrenia and delirious } \\
\text { disorders: } 150(20.4 \%) \\
\text { Substance abuse: } 85 \\
(11.2 \%)\end{array}$ & $\begin{array}{l}\text { Suicide attempts } \\
\text { were two times more } \\
\text { frequent among } \\
\text { women than among } \\
\text { men. Young people, } \\
\text { both men and women. }\end{array}$ & $\begin{array}{l}\text { Patients hospitalized in } \\
\text { general wards and not } \\
\text { in psychiatric wards. } \\
\text { No control group. }\end{array}$ \\
\hline
\end{tabular}




\begin{tabular}{|c|c|c|}
\hline $\begin{array}{l}\text { Lee et al., } \\
2012^{29}\end{array}$ & $\begin{array}{l}\text { To describe risk factors of } \\
\text { attempted suicide associated with } \\
\text { intensity of self-inflicted injuries. }\end{array}$ & $\begin{array}{l}\text { Men: } p=0.001 \text { (severe } \\
\text { injury group) } \\
\text { Age: } 10 \text { to } 19 \text { years; } p= \\
0.001 \text { (severe injury group) } \\
\text { consumed no alcohol: } p= \\
0.001 \text { (severe injury group) }\end{array}$ \\
\hline $\begin{array}{l}\text { Turhan et al., } \\
2012^{30}\end{array}$ & $\begin{array}{l}\text { To determine suicide rates, risk } \\
\text { factors and reasons for suicide } \\
\text { attempts. }\end{array}$ & $\begin{array}{l}\text { Young people ( } 15-24 \text { years): } \\
87.1 \text { (odds ratio) } \\
\text { Women/men: } 3.27 \text { (odds } \\
\text { ratio) } \\
\text { Single women: } 2.7 \text { (odds } \\
\text { ratio) } \\
\text { Higher education level: } 8.4 \\
\text { (odds ratio) }\end{array}$ \\
\hline
\end{tabular}

Garcia-Rabago To identify differences between et al., 2010 31 patients with high-and lowlethality risk factors.
Yip et al., $2011^{32}$

Oh et al., $2011^{22}$

Reazie et al., $2011^{33}$

Chowdhury, et al., $2010^{34}$
To compare the characteristics of patients hospitalized in Hong Kong and Oxford after repeated suicide attempt.

To describe factors associated with repeated suicide attempts.

To compare the profile of suicide attempts by self-immolation and poisoning.

To evaluate the characteristics of people that attempted suicide and survived and those that died, seen in a primary public healthcare service
Living alone: 6.7 (odds ratio) Prior alcohol poisoning: 3.8 (odds ratio)
Single $(p=0.04)$

Victim of violence in the last

5 years $(p=0.02)$

Hospitalization $(p=0.02)$

Adverse life problems ( $p=$

0.01)

Consequences of sexual abuse in childhood ( $p=0.04$ )

Consequences of physical abuse in childhood $(p=0.01)$ Repetitive self-mutilation ( $p$ $=0.00$ )

Female sex ( $p=0.002)$

Not having a family ( $p<$ 0.001)

Attempt method: analgesics $(p<0.001)$

History of psychiatric

treatment $(p<0.001)$ of severe suicide greater among people

$53.3 \%(p<0.001)$; younger (25 years) $(p<0.007)$; single $59.9 \%$; higher educational level ( $p<0.001)$ Positive suicidal intent behavior: $44.5 \%(p<0.001)$

In both groups, risk factors were:

Age: 24 years

Women: $61.7 \%$

Illiterate: $39.5 \%$

Married: $71.9 \%$

Domestic conflicts and

violence: $46.8 \%$
Male sex, older

age and not having

consumed alcohol

were predictors

attempt.

Young people, women, single status and people with higher educational level and history of psychiatric disease in the family

had a higher risk of attempts and recurrence.

In the high-lethality group, chances of attempted suicide were 6.7 times that lived along and 3.8 times among people with a history of previous alcoholic poisoning.

Suicide intent: Hong Kong (48.9\%) and Oxford (29\%). Intent was higher among men in Oxford (35.71)

than among women (25.7). Defaulted psychiatric treatment: 44. $6 \%$ in Hong Kong and $35.3 \%$ in Oxford. Alcohol abuse: Oxford $33.2 \%$; and Hong Kong $14 \%$.

The most relevant factors were female sex, not having a family, having a history of psychiatric treatment and use of antidepressants.

Risk factors were different in the two groups.

Data may be

underestimated, as

Koreans tend to mask

their suicidal intent.

Study based on medical records. Higher educational level was a risk factor in this study.

Lethality evaluated using instruments (Insa and Barrachina scale).

Study conducted in only one hospital.

Short repetition follow-up (6 months). Large number of interviewees had a previous attempt. Authors believe more details are essential to evaluate actual risk factors and suggest

that suicide intent scales should be different for different sexes.

Data were collected from self-reports. Attempt repetitions may have been underreported due to collection at one site only. Study did not examine reasons for attempt, social class, marital status, use of substances, or other diseases.

Data from one hospital only and short study

duration.

No validated

instruments for

assessment.

Both groups were classified as an important public health problem in the Sudarban area; correct pesticide management is essential as a preventive strategy.
Cultural factors seem to have affected results, which differed from other data reported in the literature (marital status and low educational level). 


\begin{tabular}{|c|c|c|c|c|}
\hline $\begin{array}{l}\text { Zyould et al., } \\
2010^{24}\end{array}$ & $\begin{array}{l}\text { To determine risk factors and life } \\
\text { stressors that predominate in } \\
\text { cases of deliberate self-poisoning } \\
\text { (DSP) with acetaminophen and } \\
\text { identify gender differences in } \\
\text { associated factors, as well as } \\
\text { to determine the prevalence } \\
\text { of psychiatric diagnoses and } \\
\text { the patterns and types of } \\
\text { psychotherapeutic interventions } \\
\text { provided by psychiatrists. }\end{array}$ & $\begin{array}{l}\text { Risk factor for men: } \\
\text { Chronic alcohol intake }(p= \\
0.04) \\
\text { Higher reported dose } \\
\text { ingested }(p=0.01) \\
\text { Higher latency time ( } p= \\
0.04) \\
\text { Length of hospital stay ( } p= \\
0.03)\end{array}$ & $\begin{array}{l}\text { There were no } \\
\text { significant differences } \\
\text { between sexes. Men } \\
\text { had greater toxicity } \\
\text { because of greater } \\
\text { chronic use of alcohol, } \\
\text { greater doses and } \\
\text { longer latency time. }\end{array}$ & $\begin{array}{l}\text { Specific findings for } \\
\text { male sex, particularly } \\
\text { associated with alcohol } \\
\text { intake and DSP with } \\
\text { acetaminophen. } \\
\text { Psychiatric diagnoses } \\
\text { made according to } \\
\text { clinical examination } \\
\text { and not by means of } \\
\text { structured instruments. }\end{array}$ \\
\hline $\begin{array}{l}\text { Heyerdahl et } \\
\text { al., } 2010^{35}\end{array}$ & $\begin{array}{l}\text { To investigate differences in } \\
\text { psychosocial factors and referrals } \\
\text { to follow-up among self-poisoned } \\
\text { patients according to intention. }\end{array}$ & $\begin{array}{l}\text { Age: } 30 \text { to } 49 \text { years; OR: } \\
0.51 \text { ( } 95 \% \text { CI } 0.34-0.77) \\
\text { Immigrant from Asian } \\
\text { countries, OR: } 0.23 \text { ( } 95 \% \text { CI } \\
0.11-0.49) \\
\text { Living alone, OR: } 2.21 \text { ( } 95 \% \\
\text { CI } 1.12-4.37) \\
\text { Sick leave, OR: } 0.30 \text { (95\% CI } \\
0.15-0.61)\end{array}$ & $\begin{array}{l}\text { Considerable } \\
\text { similarities in social } \\
\text { deprivation, drug use, } \\
\text { previous attempts } \\
\text { and previous or } \\
\text { current psychiatric } \\
\text { treatment between } \\
\text { groups of attempted } \\
\text { suicide and appealing } \\
\text { attempts. The drug or } \\
\text { alcohol misuse group } \\
\text { had no referral after } \\
\text { discharge and seemed } \\
\text { to be excluded from } \\
\text { follow-up, although } \\
\text { their risk is well } \\
\text { known. }\end{array}$ & $\begin{array}{l}\text { No validated } \\
\text { instruments for } \\
\text { assessment. } \\
\text { Relevance of findings } \\
\text { stress importance of } \\
\text { physical examination. } \\
\text { Drug and alcohol } \\
\text { misusers (risk group) } \\
\text { were not referred to } \\
\text { treatment. }\end{array}$ \\
\hline $\begin{array}{l}\text { Ozdel et al., } \\
2009^{36}\end{array}$ & $\begin{array}{l}\text { To examine the sociodemographic } \\
\text { and clinical characteristics of a } \\
\text { sample of } 144 \text { suicide attempters } \\
\text { in an emergency clinic for suicide } \\
\text { attempts. }\end{array}$ & $\begin{array}{l}\text { Female sex: } 75 \% \\
\text { Low educational level ( } p= \\
0.05 \text { ) } \\
\text { Low religious orientation ( } p \\
=0.02 \text { ) }\end{array}$ & $\begin{array}{l}\text { Suicide attempts were } \\
\text { more frequent among } \\
\text { internal immigrant } \\
\text { women with a low } \\
\text { educational level } \\
\text { and low religious } \\
\text { orientation; the } \\
\text { main method was } \\
\text { drug overdose, and } \\
\text { the main cause, } \\
\text { depression due to } \\
\text { conflict within the } \\
\text { family. }\end{array}$ & $\begin{array}{l}\text { Low educational level } \\
\text { was a risk factor of } \\
\text { attempted suicides. } \\
\text { No control group. }\end{array}$ \\
\hline $\begin{array}{l}\text { Payne et al., } \\
2009^{37}\end{array}$ & $\begin{array}{l}\text { To identify factors that influence } \\
\text { hospital readmission due to self- } \\
\text { poisoning. }\end{array}$ & $\begin{array}{l}\text { Risk factors for readmission: } \\
\text { Previous psychiatric hospital } \\
\text { admission (OR } 2.85, \mathrm{p}< \\
0.01 \text { ) } \\
\text { Personality disorder (OR } \\
4.59, \mathrm{p}<0.01 \text { ) } \\
\text { Increased deprivation } \\
\text { (quintile } 3 \text { : OR } 1.6, \mathrm{p}<0.01 \text {; } \\
\text { quintile } 5: \text { OR } 1.15, \mathrm{p}<0.01 \text {, } \\
\text { compared with quintile } 1 \text { ) } \\
\text { Taking medicines for chronic } \\
\text { disease, drug dependency } \\
\text { (OR } 1.6 \text { and } 1.19, \mathrm{p} \leq 0.02 \text { ) } \\
\text { Medication dependency }(\mathrm{OR} \\
1.6 \text { and } 1.19, \mathrm{p} \leq 0.02 \text { ) } \\
\text { Use of antidepressants } \\
\text { (compared with paracetamol) } \\
\text { (OR } 1.11, \mathrm{p}=0.01 \text { ) } \\
\text { Coingestion of three or more } \\
\text { agents }(\mathrm{OR} 1.37, \mathrm{p}<0.01 \text { ) }\end{array}$ & $\begin{array}{l}\text { Younger age, greater } \\
\text { deprivation (quintile), } \\
\text { ingestion of certain } \\
\text { groups of drugs } \\
\text { or multiple drug } \\
\text { types and previous } \\
\text { hospital admission } \\
\text { were risk factors for } \\
\text { readmission due to } \\
\text { self-poisoning. }\end{array}$ & $\begin{array}{l}\text { The study did not } \\
\text { provide an analysis } \\
\text { of the effects of } \\
\text { drug dependency on } \\
\text { readmission rates, } \\
\text { a factor that has } \\
\text { been associated with } \\
\text { recurrent self-harm. } \\
\text { Study did not evaluate } \\
\text { suicide intent. }\end{array}$ \\
\hline
\end{tabular}




\begin{tabular}{|c|c|c|c|c|}
\hline $\begin{array}{l}\text { Carter et al., } \\
2007^{38}\end{array}$ & $\begin{array}{l}\text { To determine whether similar } \\
\text { social and psychiatric factors are } \\
\text { associated with suicide attempts } \\
\text { in community and clinical } \\
\text { settings, and whether magnitude } \\
\text { of effect is greater in clinical } \\
\text { populations. }\end{array}$ & 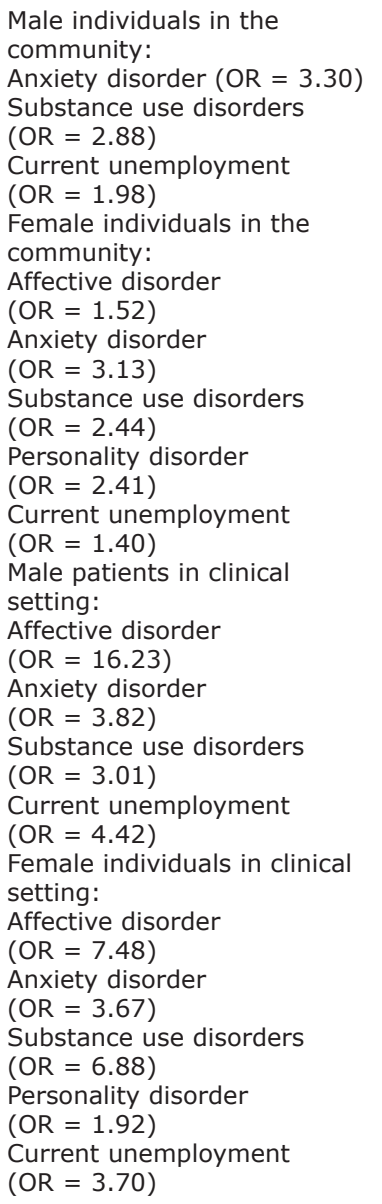 & $\begin{array}{l}\text { Magnitude of risk } \\
\text { factors among clinical } \\
\text { cases was equal to } \\
\text { or greater than that } \\
\text { among community } \\
\text { cases. }\end{array}$ & $\begin{array}{l}\text { The study suggests } \\
\text { that public health } \\
\text { interventions should } \\
\text { be aimed at modifiable } \\
\text { psychosocial factors, } \\
\text { such as education, } \\
\text { income and psychiatric } \\
\text { treatment. }\end{array}$ \\
\hline $\begin{array}{l}\text { Kapur et al., } \\
2006^{4}\end{array}$ & $\begin{array}{l}\text { To determine the proportion of } \\
\text { individuals that repeated suicide } \\
\text { attempt within } 12 \text { months, to } \\
\text { investigate timing of repetition } \\
\text { and risk factors associated with } \\
\text { repetition and their population } \\
\text { impact. }\end{array}$ & $\begin{array}{l}\text { Age }>54 \text { years }(p<0.01) \\
\text { Widowed ( } p<0.01) \\
\text { Current psychiatric treatment } \\
(p<0.01) \\
\text { Psychiatric treatment more } \\
\text { than } 12 \text { months before }(p< \\
0.01) \\
\text { Alcohol use ( } p<0.01) \\
\text { Childhood abuse }(p<0.01) \\
\text { Problems in relationships with } \\
\text { friends ( }<0.01) \\
\text { Response to mental problems } \\
\text { ( } p<0.01 \text { ) } \\
\text { Index episode } \\
\text { (premeditation) ( }<<0.01) \\
\text { Attempt method (presence } \\
\text { and suicidal behavior) } \\
\text { (drowning or asphyxiation) ( } p \\
<0.01 \text { ) }\end{array}$ & $\begin{array}{l}\text { Risk factors for } \\
\text { repetition of suicide } \\
\text { attempt were } \\
\text { psychiatric treatment, } \\
\text { being unemployed or } \\
\text { registered sick, self- } \\
\text { injury, alcohol misuse } \\
\text { and reporting suicidal } \\
\text { plans or hallucinations } \\
\text { at the time of } \\
\text { index episode. The } \\
\text { combined population } \\
\text { attributable fraction } \\
\text { (an indicator of the } \\
\text { potential population } \\
\text { impact) for these } \\
\text { variables was } 65 \% \text {. }\end{array}$ & $\begin{array}{l}\text { Study was part of } \\
\text { the Manchester and } \\
\text { Saltford Self-Harm } \\
\text { Project (MASSH) } \\
\text { of the University of } \\
\text { Manchester. } \\
\text { Study did not evaluate } \\
\text { suicidal intent; } \\
\text { Not all cases of } \\
\text { repetition were } \\
\text { included (patients seen } \\
\text { in other hospitals); } \\
\text { "Suicidal behavior" } \\
\text { equal to "self-harm", } \\
\text { both defined as an } \\
\text { "act of intentional } \\
\text { self-poisoning or injury } \\
\text { irrespective of the } \\
\text { apparent purpose of } \\
\text { the act." This definition } \\
\text { is similar to the terms } \\
\text { "attempted suicide" } \\
\text { and "parasuicide" used } \\
\text { in several countries. }\end{array}$ \\
\hline
\end{tabular}


Carter et al., $2006^{39}$

Alaghehbandan et al., $2005^{40}$

Ichimura et al. $2005^{4}$
To describe risk factors of referral Age 25-34 (1.61:1.22-

to psychiatric hospitalization after hospitalization in general hospital due to self-poisoning in an Australian sample.

To examine epidemiology and factors associated with suicide attempts requiring hospitalization in the province of Newfoundland and Labrador.

To clarify characteristics of depression and measures to prevent suicide due to depression in comparison with other mental disorders.
$2.14)$ and 35 years or older

(1.94:1.44-2.59)

Divorced or separated

(0.60:0.46-78)

High school education

(1.31:0.74-2.32)

Homelessness (3.29:1.82-

5.03) Unemployment

(1.39:1.04-1.86) Self-injury

(1.35:1.11-1.65) Psychiatric hospitalization within

12 months (2.63:2.06

3.35) Previous psychiatric

treatment (1.92:1.54-2.40)

Low to moderate suicida

ideation (4.97:3.83-6.43)

Suicidal planning (39.72:

29.05-54.33) Mood

disorders (2.22:1.69-2.92)

Adjustment disorder $(0.83$ :

0.49-1.41) Other attention

disorders (0.85: 0.67-

1.01) Schizophrenia and

other psychotic disorders

(4.68:3.00-7.30)

Female sex when individuals

in Labrador were compared

with those in Newfoundland

$(p<0.001)$

Younger age (15-24 years)

when individuals in Labrado

were compared with those

in the island portion of the

province (teen or young

adult) $(\mathrm{p}<0.001)$

Single status $(p<0.001)$

Less than 12 years of

education ( $p<0.001)$

Suicide attempt rates

among single individuals in

Labrador was higher than in

Newfoundland ( $p-0.001)$

Greater rates of mental

disorder in Newfoundland ( $p$

$<0.001$ ) Lower mean age at

time of attempt in Labrador

than in Newfoundland

$(p<0.01)$ Women in

Labrador were younger

than in Newfoundland ( $p$

$<0.01$ ) Both men and

women were significantly

younger in Labrador than in

Newfoundland ( $p<0.001$ )

Mean age between groups

49.2 vs. 33.2 ( $p<0.001$ )

Female sex between groups:

$(p<0.001)$

Divorce between groups: ( $p$

$<0.01$ )

Other attempt methods: ( $p$

$<0.01$ )

Less severe physical injury

between groups: $(p<0.01)$

Older patients: $(p<0.001)$

Odds: 0.953 (0.933-0.934);

Married or cohabiting: ( $p$ <

0.004)

Odds: 0.351 (0.173-0.714)

Methods other than

poisoning: $(p<0.007)$

Odds: $0.161(0.043-0.601)$
Psychiatric

hospitalization referral

after a suicide attempt

is a complex decision

that should take

into consideration

ideation, planning,

psychiatric history

and sociodemographic

variables.

Differences between suicide attempt rates in Newfoundland and Labrador probably

reflect social economic and cultural factors that cannot be fully evaluated in studies using secondary data.

In this study, risk factors of patients with depression were different from those of patients that attempted suicide and similar to those of individuals that committed suicide.
Analysis in a single geographic area

No structured instruments to evaluate ideation, planning and menta disorder.

Comparison between two samples (city and province)
Data analyzed in a single hospital No semi-structured interviews were used to diagnose suicide attempts;

No firm criterion to define injury severity. 


\begin{tabular}{|c|c|c|c|c|}
\hline $\begin{array}{l}\text { Fekete et al., } \\
2005^{42}\end{array}$ & $\begin{array}{l}\text { To determine differences of } \\
\text { suicidal behavior between sexes } \\
\text { and investigate factors associated } \\
\text { with suicide attempts. }\end{array}$ & $\begin{array}{l}\text { Female sex characterized by: } \\
\text { Retired or economically } \\
\text { inactive }(O R=2.38) \\
\text { Widowed }(O R=6.55) \\
\text { Divorced }(O R=1.64) \\
\text { Depression in personal } \\
\text { history }(O R=1.27)\end{array}$ & $\begin{array}{l}\text { There are significant } \\
\text { differences in risk } \\
\text { factors of suicide } \\
\text { attempts between } \\
\text { men and women. } \\
\text { These factors } \\
\text { should be taken } \\
\text { into consideration } \\
\text { in treatment and } \\
\text { prevention. }\end{array}$ & $\begin{array}{l}\text { Data only from } \\
\text { patients that received } \\
\text { medication, and } \\
\text { inclusion of patients } \\
\text { with more than one } \\
\text { attempt. }\end{array}$ \\
\hline $\begin{array}{l}\text { Douglas et al., } \\
2004^{43}\end{array}$ & $\begin{array}{l}\text { To describe clinical and } \\
\text { demographic characteristics } \\
\text { of near-fatal suicide attempts } \\
\text { (NFDSH); } \\
\text { To determine whether age and } \\
\text { sex profile of NFDSH cases is } \\
\text { similar to that of cases of suicide; } \\
\text { To describe clinical evaluation of } \\
\text { NFDSH group; } \\
\text { To collect data about individuals } \\
\text { in the NFDSH group, including } \\
\text { their views on prevention. }\end{array}$ & $\begin{array}{l}\text { Risk factors in both groups: } \\
\text { Not married: Odds: } 1.63 \\
(1.04-2.54) \\
\text { Wanted to die: Odds: } 2.11 \\
(1.40-3.18) \\
\text { Previous psychiatric } \\
\text { treatment: Odds: } 11.70 \\
\text { (1.17-2.46) } \\
\text { Previous self-harm: Odds: } \\
1.82 \text { ( } 1.24-2.66) \\
\text { Feeling depressed: Odds: } \\
2.24 \text { ( } 1.36-3.68) \\
\text { Eating disorders: Odds: } 1.69 \\
(1.16-2.47)\end{array}$ & $\begin{array}{l}\text { NFDSH cases are } \\
\text { important clinical } \\
\text { phenomena associated } \\
\text { with indicators of high } \\
\text { risk of suicide. Their } \\
\text { study may contribute } \\
\text { to suicide prevention. }\end{array}$ & $\begin{array}{l}\text { Clinical conditions of } \\
\text { patients prevented } \\
\text { further questioning; } \\
\text { NFDSH definition } \\
\text { was based on initial } \\
\text { evaluations; } \\
\text { Other NFDSH } \\
\text { definitions may be } \\
\text { used. } \\
\text { Suicidal intent was not } \\
\text { evaluated. } \\
\text { Cases of overdose, if } \\
\text { excluded, might have } \\
\text { changed results. }\end{array}$ \\
\hline $\begin{array}{l}\text { Carter et al., } \\
2003^{44}\end{array}$ & $\begin{array}{l}\text { To explore the differences } \\
\text { between a clinical sample } \\
\text { of deliberate self-poisoning } \\
\text { patients and a community } \\
\text { sample that reported previous } \\
\text { suicide attempts. To examine } \\
\text { correlates of suicidal behavior in } \\
\text { these groups compared with a } \\
\text { community control group (CC) } \\
\text { with no suicidal behavior. }\end{array}$ & $\begin{array}{l}\text { In the clinical group: } \\
\text { Female sex }(\mathrm{OR}=5.7 ; \mathrm{CI}= \\
1.7-19.4) \\
\text { Anxiety }(\mathrm{OR}=7.4 ; \mathrm{CI}= \\
2.2-25.1) \\
\text { Affective disorder }(\mathrm{OR}= \\
23.0 ; \mathrm{CI}=6.9-76.5) \\
\text { Substance use disorders (OR } \\
=19.2 ; \mathrm{CI}=5.6-65.4) \\
\text { Mental health related } \\
\text { disability (OR =0.5; CI }= \\
0.3-0.7) \\
\text { In the community group: } \\
\text { Anxiety (OR }=9.4 ; \mathrm{CI}= \\
1.7-52.8) \\
\text { Substance use disorders }(\mathrm{OR} \\
=3.0 ; \mathrm{CI}=1.1-8.7) \\
\text { Mental health related } \\
\text { disability }(\mathrm{OR}=0.5 ; \mathrm{CI}= \\
0.4-0.7) \\
\text { Affective disorder }(\mathrm{OR}=4.0 ; \\
\text { CI = 0.9-17.1) }\end{array}$ & $\begin{array}{l}\text { Correlates of } \\
\text { deliberate self- } \\
\text { poisoning were usually } \\
\text { more powerful in the } \\
\text { clinical group, but had } \\
\text { a similar pattern of } \\
\text { psychiatric disorders } \\
\text { and disability factors } \\
\text { in both groups. }\end{array}$ & $\begin{array}{l}\text { This study stresses } \\
\text { the importance of } \\
\text { analysis of modifiable } \\
\text { risk factors and } \\
\text { interventions based on } \\
\text { them } \\
\text { According to authors, } \\
\text { main limitation was } \\
\text { the memory bias in the } \\
\text { group that attempted } \\
\text { suicide at any time in } \\
\text { life. }\end{array}$ \\
\hline
\end{tabular}

Poisoning is globally accepted as the most common method of suicide attempt, which is confirmed by data reported by WHO and EURO. ${ }^{47-50}$

Table 2 describes the studies according to objectives, risk factors, main conclusions and comments. Although the studies had different objectives, they all described the characteristics or profiles of their samples.

Several explanations were provided for including sex as a risk factor, and one of them was its association with other risk factors, such as unemployment, which is an important social stressor, and marital conflicts ${ }^{51}$ and divorce, ${ }^{38}$ some of the typical stressors of life.

The greatest incidence of suicide attempts was found in the group of individuals 15 to 40 years old. Similar data were found in a multi-center study conducted in Switzerland, which reported that suicide attempts by poisoning were more common among teens and young adults. ${ }^{26}$
A recent consensus statement, prepared in 2009 and published in 2010 by several specialists in the study of suicide and suicide attempts, stresses that suicide attempts by adolescents may be associated with suicide ideation or impulsivity, but suicide in this age group is rare when compared with rates for the elderly. ${ }^{5}$

The analysis of marital status revealed that it might be both a protective and a risk factor for suicide and suicide attempts, ${ }^{52}$ as this variable is strongly affected by associated factors. Family conflicts seem to be a trigger for suicide attempts even among married women. ${ }^{34}$ Moreover, divorced and separated women had a rate of suicide twice as great as that for married women. ${ }^{53}$

Data published by the American Psychiatric Association (APA $)^{45}$ have drawn attention to the importance of several social factors, as single people may have a limited kinship and social support network. Isolation may predispose to the triggering of events 
associated with vulnerability and, consequently, possible suicide. Feelings of loneliness are associated with a greater risk of suicide attempts, and family and social support are, in general, protective factors. ${ }^{5,22,38}$

Both unemployment and low educational level have been found to be significant risk factors of suicide attempts, as they are associated with social disadvantages that may pose a greater risk of suicidal behavior. $^{54}$

The data collected in this review support studies that found that psychiatric comorbidities, such as depression and generalized anxiety disorder, or depression and oppositional defiant disorder, increase the risk of suicide attempts, particularly among younger individuals. ${ }^{37}$ Some studies found that the use of drugs and alcohol abuse were important risk factors. ${ }^{28,29,31}$

Depression was one of the most relevant risk factors associated with suicide in almost all studies. This disease, taken by its own symptoms or together with other factors, usually involves physical and psychological distress, life terminality, social, economic and cultural problems, losses, abandonment, loneliness and family conflicts. ${ }^{55}$ The powerful association between suicide and depression demands that greater attention should be paid to the diagnosis of this mental disorder, ${ }^{41}$ particularly in primary healthcare, and to the interventions designed to treat is, as important measures to prevent suicide. ${ }^{56}$

Of all studies included in this review, only the one conducted by Yip et al. found that previous self-harm was a risk factor of repeated suicide attempt. ${ }^{32}$

\section{Conclusion}

According to the results reported here, the main risk factors of suicide attempts by self-poisoning are female sex, age 15 to 40 years, single status, low educational level, unemployment, drug or alcohol abuse or addiction and psychiatric disorder, which is the most relevant factor.

Further investigations should be conducted, such as case-control studies to confirm current findings and identify new risk factors. Their results may contribute to planning interventions to prevent suicide attempts.

\section{References}

1. Bastos $\mathrm{O}$. Comportamentos suicidas em uma unidade psiquiátrica de um hospital universitário [tese de livre-docência]. Recife: Universidade Federal de Pernambuco; 1975.

2. Organização Mundial de Saúde. Prevenção do suicídio: manual dirigido a profissionais das equipes de saúde mental [on-line]. Campinas: OMS/OPAS/Unicamp; 2006. http://bvsms.saude.gov. br/bvs/publicacoes/manual_editoracao.pdf

3. Stengel E. Suicide and attempted suicide. Oxford, England: Penguin Books; 1964.

4. Kapur N, Cooper J, King-Hele S, Webb R, Lawlor M, Rodway C, et al. The repetition of suicidal behavior: a multicenter cohort study. J Clin Psychiatry. 2006;67:1599-609.
5. Meyer RE, Salzman C, Youngstrom EA, Clayton PJ, Goodwin FK, Mann JJ, et al. Suicidality and risk of suicide--definition, drug safety concerns, and a necessary target for drug development: a brief report. J Clin Psychiatry. 2010;71:1040-6. Epub 2010 Jul 13.

6. Botega NJ, Marín-León L, Oliveira HB, Barros MB, Silva VF, Dalgalarrondo P. [Prevalence of suicidal ideation, suicide plans, and attempted suicide: a population-based survey in Campinas, Sao Paulo State, Brazil]. Cad Saude Publica. 2009;25:2632-8.

7. Bando $\mathrm{DH}$, Volpe FM. Seasonal Variation of Suicide in the City of Sao Paulo, Brazil, 1996-2010. Crisis. 2013;35:5-9.

8. Bochner R. Informação sobre intoxicações e envenenamentos para a gestão do SUS: um panorama do Sistema Nacional de Informações Tóxico-Farmacológicas - SINITOX. RECIIS. 2013;7. http://www.reciis.icict.fiocruz.br/index.php/reciis/article/ viewArticle/767/1567. Accessed 2013 Aug 13.

9. Vidal CE, Gontijo EC, Lima LA. Tentativas de suicídio: fatores prognósticos e estimativa do excesso de mortalidade. Cad Saude Publica. 2013;29:175-87.

10. Rapeli CB, Botega NJ. Clinical profiles of serious suicide attempters consecutively admitted to a university-based hospital: a cluster analysis study. Rev Bras Psiquiatr. 2005;27:285-9. Epub 2005 Dec 12.

11. Stefanello S, Cais CF, Mauro ML, Freitas GV, Botega NJ. Gender differences in suicide attempts: preliminary results of the multisite intervention study on suicidal behavior (SUPRE-MISS) from Campinas, Brazil. Rev Bras Psiquiatr. 2008;30:139-43. Epub 2007 Dec 20.

12. Bernardes SS, Turini CA, Matsuo T. Perfil das tentativas de suicídio por sobredose intencional de medicamentos atendidas por um Centro de Controle de Intoxicações do Paraná, Brasil. Cad Saude Publica. 2010;26:1366-72.

13. Marín-León L, Barros MB. Mortes por suicídio: diferenças de gênero e nível socioeconômico. Rev Saúde Pública. 2003;37:357-63.

14. Viana GN, Zenkner FM, Sakae TM, Escobar BT. Prevalência de suicídio no Sul do Brasil, 2001-2005. J Bras Psiquiatr. 2008;57:38-43.

15. Baptista $M N$, Borges A. Suicídio: aspectos epidemiológicos em Limeira e adjacências no período de 1998 a 2002. Estud Psicol (Campinas). 2005;22:425-31.

16. Cibis A, Mergl R, Bramesfeld A, Althaus D, Niklewski G, Schmidtke $A$, et al. Preference of lethal methods is not the only cause for higher suicide rates in males. J Affect Disord. 2012;136:9-16. Epub 2011 Sep 19

17. Mello MF, Mello AAF, Kohn R. Epidemiologia da Saúde Mental no Brasil. Porto Alegre: Artmed; 2007.

18. Pires MC, Raposo MC, Pires M, Sougey EB, Bastos Filho OC. Stressors and attempted suicide by poisoning: a sex comparison. Trends Psychiatry Psychother. 2012;34:25-30.

19. Bortoletto ME, Bochner R. Impacto dos medicamentos nas intoxicações humanas no Brasil. Cad Saúde Pública. 1999; 15:859-69.

20. Neto AC, Gauer GJC, Furtado NR. Psiquiatria para estudantes de medicina. Porto Alegre: Edipucrs; 2003.

21. Botega N, Bertolote J, Bessa M, Hetem L. Prevenção do suicídio. Debates Psiquiatr Hoje. 2010;1:14-6.

22. Oh SH, Park KN, Jeong SH, Kim HJ, Lee CC. Deliberate selfpoisoning: factors associated with recurrent self-poisoning. Am J Emerg Med. 2011;29:908-12.

23. Gwini SM, Shaw D, Iqbal M, Spaight A, Siriwardena AN. Exploratory study of factors associated with adverse clinical features in patients presenting with non-fatal drug overdose/selfpoisoning to the ambulance service. Emerg Med J. 2011;28:8924. Epub 2010 Sep 15.

24. Zyoud SH, Awang R, Sulaiman SA, Al-Jabi SW. A cross-sectional observation of the factors associated with deliberate selfpoisoning with acetaminophen: impact of gender differences and psychiatric intervention. Hum Psychopharmacol. 2010;25:500-8.

25. Pettit JW, Paukert AL, Joiner TE Jr, Rudd MD. Pilot sample of very early onset bipolar disorder in a military population moderates the association of negative life events and non-fatal suicide attempt. Bipolar Disord. 2006;8:475-84.

26. Flavio M, Martin E, Pascal B, Stephanie C, Gabriela S, Merle K, et al. Suicide attempts in the county of Basel: results from the WHO/EURO Multicentre Study on Suicidal Behaviour. Swiss Med Wkly. 2013;143:w13759.

27. Williams-Johnson J, Williams E, Gossell-Williams M, Sewell CA, Abel WD, Whitehorne-Smith PA. Suicide attempt by selfpoisoning: characteristics of suicide attempters seen at the Emergency Room at the University Hospital of the West Indies. West Indian Med J. 2012;61:526-31. 
28. Rančić N1, Ignjatović Ristić D, Radovanović S, Kocić S, Radević S. Sociodemographic and clinical characteristics of hospitalized patients after suicide attempt: a twenty-year retrospective study. Med Glas (Zenica). 2012;9:350-5.

29. Lee CA, Choi SC, Jung KY, Cho SH, Lim KY, Pai KS, et al. Characteristics of patients who visit the emergency department with self-inflicted injury. J Korean Med Sci. 2012;27:307-12. Epub 2012 Feb 23.

30. Turhan E, Inandi T, Aslan M, Zeren C. Epidemiology of attempted suicide in Hatay, Turkey. Neurosciences (Riyadh). 2011;16:34752.

31. García-Rábago H, Sahagún-Flores JE, Ruiz-Gómez A, SánchezUreña GM, Tirado-Vargas JC, González-Gámez JG. Factores de riesgo, asociados a intento de suicidio, comparando factores de alta y baja letalidad. Rev Salud Pública. 2010;12:713-21.

32. Yip PS, Hawton K, Liu K, Liu KS, Ng PW, Kam PM, et al. A study of deliberate self-harm and its repetition among patients presenting to an emergency department. Crisis. 2011;32:217-24.

33. Rezaie L, Khazaie $H$, Soleimani A, Schwebel DC. Is self-immolation a distinct method for suicide? A comparison of Iranian patients attempting suicide by self-immolation and by poisoning. Burns. 2011;37:159-63. Epub 2010 Aug 13.

34. Chowdhury AN, Banerjee $S$, Brahma A, Das $S$, Sarker $P$, Biswas $M K$, et al. A prospective study of suicidal behaviour in Sundarban Delta, West Bengal, India. Natl Med J India. 2010;23:201-5.

35 Heyerdahl F, Bjornaas MA, Dahl R, Hovda KE, Nore AK, Ekeberg $\mathrm{O}$, et al. Repetition of acute poisoning in Oslo: 1 -year prospective study. Br J Psychiatry. 2009;194:73-9.

36. Ozdel O, Varma G, Atesci FC, Oguzhanoglu NK, Karadag F, Amuk T. Characteristics of suicidal behavior in a Turkish sample. Crisis. 2009;30:90-3.

37. Payne RA, Oliver JJ, Bain M, Elders A, Bateman DN. Patterns and predictors of re-admission to hospital with self-poisoning in Scotland. Public Health. 2009;123:134-7. Epub 2009 Jan 30.

38. Carter GL, Child C, Page A, Clover K, Taylor R. Modifiable risk factors for attempted suicide in Australian clinical and community samples. Suicide Life Threat Behav. 2007;37:671-80.

39. Carter GL, Safranko I, Lewin TJ, Whyte IM, Bryant JL. Psychiatric hospitalization after deliberate self-poisoning. Suicide Life Threat Behav. 2006;36:213-22.

40. Alaghehbandan R, Gates KD, MacDonald D. Suicide attempts and associated factors in Newfoundland and Labrador, 1998-2000. Can J Psychiatry. 2005;50:762-8.

41. Ichimura $A$, Matsumoto $H$, Aoki $T$, Andoh $H$, Yano $H$, Nakagawa Y, et al. Characteristics of suicide attempters with depressive disorders. Psychiatry Clin Neurosci. 2005;59:590-4.

42. Fekete S, Voros V, Osvath P. Gender differences in suicide attempters in Hungary: retrospective epidemiological study. Croat Med J. 2005;46:288-93.

43. Douglas J, Cooper J, Amos T, Webb R, Guthrie E, Appleby L. "Near-fatal" deliberate self-harm: characteristics, prevention and implications for the prevention of suicide. J Affect Disord. 2004; 79:263-8.

44. Carter GL, Issakidis C, Clover K. Correlates of youth suicide attempters in Australian community and clinical samples. Aust $\mathrm{N}$ Z J Psychiatry. 2003;37:286-93.

45. Association AP. Diretrizes para o tratamento de transtornos psiquiátricos-Compêndio 2006. Porto Alegre: Artmed. 2006.

46. Elkis $H$. Fatores de impacto de publicações psiquiátricas e produtividade científica. Rev Bras Psiquiatr. 1999;21:231-6.

47. Arantes-Gonçalves F, Coelho R. À procura de marcadores biológicos no comportamento suicidário. Acta Med Port. 2008;21:89-98.

48. Schmidtke $A$, Bille-Brahe $U$, DeLeo $D$, Kerkhof $A$, Bjerke $T$, Crepet $\mathrm{P}$, et al. Attempted suicide in Europe: rates, trends and sociodemographic characteristics of suicide attempters during the period 1989-1992. Results of the WHO/EURO Multicentre Study on Parasuicide. Acta Psychiatr Scand. 1996;93:327-38.

49. Bogdanovica I, Jiang GX, Lohr C, Schmidtke A, MittendorferRutz E. Changes in rates, methods and characteristics of suicide attempters over a 15-year period: comparison between Stockholm, Sweden, and Würzburg, Germany. Soc Psychiatry Psychiatr Epidemiol. 2011;46:1103-14. Epub 2010 Sep 5.

50. Gunnell D, Eddleston M. Suicide by intentional ingestion of pesticides: a continuing tragedy in developing countries. Int J Epidemiol. 2003;32:902-9.

51. Maris RW. Social forces in urban suicide. Dorsey Press; 1969.

52. Qin $P$, Agerbo $E$, Mortensen PB. Suicide risk in relation to socioeconomic, demographic, psychiatric, and familial factors: a national register-based study of all suicides in Denmark, 19811997. Am J Psychiatry. 2003;160:765-72.

53. Luoma JB, Martin CE, Pearson JL. Contact with mental health and primary care providers before suicide: a review of the evidence. Am J Psychiatry. 2002;159:909-16.

54. Nock MK, Borges G, Bromet EJ, Cha CB, Kessler RC, Lee S. Suicide and suicidal behavior. Epidemiol Rev. 2008;30:133-54. Epub 2008 Jul 24.

55. Minayo MC, Cavalcante FG. Suicídio entre pessoas idosas: revisão da literatura. Rev Saúde Pública. 2010;44:750-7.

56. Beeston D, Partnership CSI, Ageing Cf, Mental Health SU. Older People and Suicide. Centre for Ageing and Mental Health, Staffordshire University; 2006.

\section{Correspondence:}

Maria Cláudia da Cruz Pires

Universidade Federal de Pernambuco

Rua das Pernambucanas, 282, sala 305, Graças

52011-010 - Recife, PE - Brazil

Tel./Fax: +55 (81) 9469.4387

E-mail: claudiacpires@globo.com 